\title{
Die begrenzten „war powers“ des Bundestags
}

\section{Klaus Brummer}

Die Streitkräfte westlicher Staaten werden derzeit vor allem in multinationalen Missionen zum Krisen- und Konfliktmanagement eingesetzt. In Afghanistan weist die NATO-geführte International Security Assistance Force (ISAF) noch immer eine Stärke von rund 50.000 Soldatinnen und Soldaten auf. ${ }^{1}$ Hinzu kommen circa 3.200 Kräfte, die in EU-Militärmissionen aktiv sind, sowie etwa 5.100 Soldatinnen und Soldaten aus den Mitgliedstaaten von NATO und EU, die an Blauhelmmissionen der Vereinten Nationen (VN) beteiligt sind. ${ }^{2}$ Nicht zuletzt aufgrund der Größenordnung der Einsätze ist nach ihrer demokratischen Legitimierung zu fragen. Dies gilt auch für Deutschland, das zu den größten Truppenstellern gerade in NATO- und EU-Missionen zählt - derzeit sind mehr als 4.200 Kräfte der Bundeswehr im Auslandseinsatz. ${ }^{3}$

Der Bundestag gilt bei Entscheidungen zur Entsendung der Bundeswehr in Auslandseinsätze als eines der „stärksten“ Parlamente weltweit. Gleichwohl scheint er schlecht positioniert zu sein, um die ihm zur Verfügung stehenden formellen wie auch informellen Wege zur Beeinflussung der Bundesregierung bei Entsendeentscheidungen - seine „war powers“ - zu nutzen. Neben den Einschränkungen, die sich aus den Kompetenzen der Bundesregierung ergeben, werden die Einflussmöglichkeiten des Bundestags durch mehrere „parlamentsinterne“ Faktoren zusätzlich begrenzt.

Die erste Begrenzung ergibt sich aus der geringen Konfliktbereitschaft der Mehrheitsfraktionen gegenüber der „eigenen“ Regierung. Sie folgt nicht zuletzt aus der Funktionslogik parlamentarischer Regierungssysteme, die durch ein „Spannungsverhältnis zwischen Regierungsmehrheit und parlamentarischer Opposition “4 geprägt ist. Da die Abgeordneten der Regierungsfraktionen auf der Grundlage ihres freien Mandats allerdings jederzeit gegen ihre Regierung stimmen könnten, weist dieser Aspekt auch auf eine „individuell“ bedingte Begrenzung der Entscheidungsspielräume der Abgeordneten hin. Die Verbindung struktureller und individueller Faktoren zeigt sich auch an den beiden anderen Handlungsgrenzen, die weder auf die Verschränkung von Regierung und Parlamentsmehrheit zurückzuführen noch auf parlamentarische Regierungssysteme begrenzt sind. Gemeint sind Uneinigkeiten sowohl innerhalb der Oppositionsfraktionen als auch fraktionsübergreifend zwischen Fachpolitikern. Zusammengenommen führen die genannten Faktoren dazu, dass die vordergründig starken formellen und informellen „war powers“ in der Praxis kaum greifen.

1 Vgl. NATO, NATO and Afghanistan, 1 June 2014, http://www.nato.int/cps/en/natolive/69772 (Abruf am 5. August 2014).

2 Vgl. European Union External Action Service, Ongoing Missions and Operations, July 2014, http://www.eeas.europa.eu/csdp/missions-and-operations (Abruf am 5. August 2014); United Nations, Monthly Summary of Contributions, 30 June 2014, http://www.un.org/en/peacekeeping/contributors/2014/jun14_1.pdf (Abruf am 5. August 2014).

3 Vgl. Bundeswehr, Einsatzzahlen - Die Stärke der deutschen Einsatzkontingente, 28. Juli 2014, http://www.bundeswehr.de (Abruf am 5. August 2014).

4 Winfried Steffani, Parlamentarische und präsidentielle Demokratie. Strukturelle Aspekte westlicher Demokratien, Opladen 1979, S. 155. 
Der Beitrag illustriert die Handlungsbegrenzungen des Bundestags anhand der von den VN geführten Mission UNIFIL im beziehungsweise vor der Küste des Libanon. Die Beteiligung der Bundeswehr an diesem Einsatz wurde 2006 von der Regierung der damaligen Großen Koalition beantragt und vom Bundestag beschlossen. Aufgrund der komfortablen Mehrheitsverhältnisse - der Regierungskoalition gehörten mehr als 72 Prozent der Abgeordneten an - waren die Spielräume für die Regierungsfraktionen ungleich größer als bei einer Koalition mit einer knappen parlamentarischen Mehrheit. Da die Mehrheit außer Frage stand, hätten die Abgeordneten besonderen Druck auf „ihre“ Regierung ausüben können, ihre Wünsche bei der Mandatsformulierung zu berücksichtigen. Zugleich konnten die Oppositionsfraktionen angesichts der klaren Mehrheitsverhältnisse nur dann auf eine Einflussnahme auf die Regierung hoffen, wenn sie geschlossen auftreten würden. Vor diesem Hintergrund hätten eine größere Konfliktbereitschaft der Regierungsfraktionen wie auch eine möglichst einheitliche Haltung der Oppositionsfraktionen nahegelegen. Dass dies nicht der Fall war und stattdessen mehrere Handlungsbegrenzungen zutage traten, könnte ein Hinweis darauf sein, wie verbreitet letztere sind - wie auch dafür, dass sie unter der neuen Großen Koalition, die sogar einen noch größeren Anteil an Bundestagsmandaten auf sich vereint, erneut auftreten dürften.

Die sich aus dem Untersuchungsgegenstand ergebende Quellenproblematik (vertrauliche Beratungen, informelle Absprachen etc.) sollte dadurch abgemildert werden, dass möglichst vielfältige empirische Quellen (Bundestagsdrucksachen, Plenarprotokolle etc.) konsultiert wurden. Hinzu kommen acht Interviews mit aktuellen oder ehemaligen Mitgliedern des Bundestags (aus den Reihen von SPD, FDP und Bündnis 90/Die Grünen) - alle waren beziehungsweise sind Mitglieder des Auswärtigen Ausschusses oder des Verteidigungsausschusses - sowie mit einem ehemaligen Referenten der Unionsfraktion, der mit außen- und sicherheitspolitischen Themen befasst war. ${ }^{5}$

\section{Parlamentarische „war powers“}

\subsection{Vergleichende Perspektiven}

Die Beteiligungsrechte von nationalen Parlamenten bei Entscheidungen zur Entsendung von Streitkräften wurden in mehreren vergleichend angelegten Studien thematisiert. Sandra Dieterich, Hartwig Hummel und Stefan Marschall untersuchten die „parliamentary war powers" von 25 europäischen Parlamenten. ${ }^{6}$ Sie unterteilten die „Machtressourcen “7 von Parlamenten in vier Kategorien: Gesetzgebungs- und Budgetkompetenzen, Kontrollrechte, Artikulations- und Kommunikationsmöglichkeiten sowie Abwahl- beziehungsweise Wahlkompetenzen. Auf dieser Grundlage entwickelten sie eine Typologie parlamentarischer „war powers“. Diese reichen von „sehr starken“ Parlamenten, deren Zustimmung zum Einsatz

5 Den Gesprächspartnern wurde Anonymität zugesichert.

6 Vgl. Sandra Dieterich / Hartwig Hummel / Stefan Marschall, Strengthening Parliamentary „War Powers" in Europe: Lessons from 25 National Parliaments, DCAF Policy Paper No. 27, Genf 2008; dies., Parliamentary War Powers in Europe: A Survey of 25 European Parliaments, DCAF Occasional Paper No. 21, Genf 2010.

7 Dies., Parliamentary War Powers in Europe, a.a.O. (Fn. 6), S. 9. 
militärischer Gewalt ex ante notwendig ist, bis hin zu „sehr schwachen“ Parlamenten, die weder dem Einsatz militärischer Gewalt zustimmen müssen noch andere Funktionen (Kontrolle etc.) ausüben können. ${ }^{8}$

Einen in geographischer Hinsicht noch breiteren Ansatz wählen Dirk Peters und Wolfgang Wagner, die sich mit den „war powers“ von nationalen Parlamenten in insgesamt 49 demokratischen Staaten auseinandersetzen. ${ }^{9}$ Dabei konzentrieren sie sich auf die verfassungsrechtlichen beziehungsweise einfachgesetzlichen Möglichkeiten des Parlaments, den Einsatz von Streitkräften durch die Exekutive zu kontrollieren. ${ }^{10}$ Auch Peters und Wagner kommen zu einem differenzierten Bild. Sie betonen, dass nur rund ein Drittel der untersuchten Parlamente ein ex ante-Vetorecht besäße. ${ }^{11}$

Auch wenn sich die beiden dargestellten Forschungszusammenhänge anhand der analytischen Kategorien sowie der untersuchten Staaten teilweise voneinander unterscheiden, sind sie sich einig in der Einordnung der „war powers“ des Deutschen Bundestages. Seine diesbezüglichen Kompetenzen werden stets in die „oberste“ Kategorie eingeordnet, verstanden als diejenige mit den weitreichendsten Mitwirkungsrechten bei Entscheidungen zur Entsendung des Militärs ins Ausland, sei es speziell mit Blick auf das ex ante-Vetorecht ${ }^{12}$, sei es hinsichtlich eines breiteren Verständnisses von parlamentarischen Beteiligungsrechten. ${ }^{13}$

Entgegen dieser auf das Potenzial des Bundestags abhebenden Wertungen wird im Folgenden allerdings argumentiert, dass dessen Einflussmöglichkeiten in der politischen Praxis in mehrfacher Hinsicht begrenzt sind. Um die Schwachpunkte einzuordnen, lässt sich auf die Arbeit von Hans Born und Heiner Hänggi zurückgreifen. ${ }^{14}$ Diese gruppieren Defizite nationaler Parlamente bei der Kontrolle von nationalen Regierungen bei Entscheidungen zu Friedensmissionen in drei Kategorien: „Authority“ verweist auf die formalen Rechte, die den Parlamenten zustehen, um die Regierung zur Rechenschaft zu ziehen; „ability“ hebt auf die Ressourcen wie auch die Expertise von Parlamenten und Parlamentariern ab, und „attitude“ bezieht sich auf die Bereitschaft der Abgeordneten, die vorhandenen Mittel auch einzusetzen.

\subsection{Die „war powers“ des Bundestags}

Der Bundestag gilt, wie angeführt, weltweit als eines der Parlamente mit den größten Kompetenzen in Fragen der Entsendung nationaler Streitkräfte in militärische Auslandseinsät-

8 Vgl. ebenda, S. 13 f.

9 Vgl. Dirk Peters / Wolfgang Wagner, Between Military Efficiency and Democratic Legitimacy: Mapping Parliamentary War Powers in Contemporary Democracies, 1989-2004, in: Parliamentary Affairs, 64. Jg. (2011), H. 1, S. 175 - 192; Wolfgang Wagner / Dirk Peters / Cosima Glahn, Parliamentary War Powers Around the World, 1989-2004. A New Dataset, DCAF Occasional Paper No. 22, Genf 2010.

10 Vgl. Dirk Peters / Wolfgang Wagner, a.a.O. (Fn. 9), S. 178.

11 Vgl. ebenda, S. 179.

12 Vgl. ebenda, S. 189.

13 Vgl. Sandra Dieterich / Hartwig Hummel / Stefan Marschall, Parliamentary War Powers in Europe, a.a.O. (Fn. 6), S. 22.

14 Vgl. Hans Born / Heiner Hänggi, The Use of Force under International Auspices: Strengthening Parliamentary Accountability, DCAF Policy Paper No. 7, Genf 2005. 
ze. ${ }^{15}$ Seine „war powers“ manifestieren sich in der Notwendigkeit der vorherigen Zustimmung zum Einsatz militärischer Gewalt wie auch in der Befähigung, diesen Einsatz zu untersuchen und zu debattieren. ${ }^{16}$

Die starke Stellung des deutschen Parlaments gründet auf dem wegweisenden Urteil des Bundesverfassungsgerichts vom Juli 1994 und dem im März 2005 in Kraft getretenen „Parlamentsbeteiligungsgesetz " (ParlBG). ${ }^{17}$ In ihrem Urteil konzipierten die Karlsruher Richter die Bundeswehr als ein „Parlamentsheer“, wodurch einzelfallbezogene Entscheidungen zur Entsendung der Bundeswehr ins Ausland an die konstitutive Zustimmung des Bundestags gekoppelt wurden. Das ParlBG legt entsprechend die Notwendigkeit der Zustimmung des Bundestags zum „Einsatz bewaffneter deutscher Streitkräfte außerhalb des Geltungsbereichs des Grundgesetzes“ ( $\$ 1$ Abs. 2 ParlBG) fest. Über die im ParlBG angelegte Mitwirkung bei Entscheidungen zu Auslandseinsätzen verfügt der Bundestag über weitere, allgemein auf die Außenpolitik bezogene Rechte, etwa Informations- oder Kontrollrechte gegenüber der Regierung, von denen er zunehmend Gebrauch macht. ${ }^{18}$

$\mathrm{Zu}$ diesen formellen Rechten kommen informelle Einflussmöglichkeiten des Parlaments hinzu, mittels derer bereits bei der Anbahnung von Regierungsentscheidungen Einfluss auf die Inhalte des Regierungsantrags genommen werden $\mathrm{kann}^{19}$ und die laut Manfred Schwarzmeier u.a. „die Rationalität von Entscheidungen“ steigern sowie „die Integration von Widerspruch und von Spannungen erleichter[n] “ helfen. ${ }^{20}$ Angesichts des Funktions-

15 Zur Rolle des Bundestags sowie dem Zusammenspiel von Bundesregierung und Bundestag bei Entscheidungen zur Entsendung der Bundeswehr in Auslandseinsätze vgl. Rafael Biermann, Der Deutsche Bundestag und die Auslandseinsätze der Bundeswehr. Zur Gratwanderung zwischen exekutiver Prärogative und legislativer Mitwirkung, in: ZParl, 35. Jg. (2004), H. 4, S. 607 - 626; Roman Schmidt-Radefeldt, Parlamentarische Kontrolle der internationalen Streitkräfteintegration, Berlin 2005; Andreas L. Paulus, Die Parlamentszustimmung zu Auslandseinsätzen nach dem Parlamentsbeteiligungsgesetz, in: Dieter Weingärtner (Hrsg.), Einsatz der Bundeswehr im Ausland. Rechtsgrundlagen und Rechtspraxis, Baden-Baden 2007, S. 81 - 113; Dieter Wiefelspütz, Auslandseinsätze deutscher Streitkräfte und der Bundestag. Ist eine Reform geboten?, in: ZParl, 39. Jg. (2008), H. 2, S. 203 - 219; Jan Ryjáček, Der Entscheidungsprozess über den Bundeswehreinsatz zum Schutz der Wahlen im Kongo, in: ZParl, 39. Jg. (2008), H. 2, S. 219 - 232; ders., Losing the Power of Parliament? Participation of the Bundestag in the Decision-Making Process Concerning Out-of-Area Military Operations, in: German Politics, 18. Jg. (2009), H. 4, S. 485 - 500; Jörn Thießen / Ulrich Plate, Bundeswehr und Parlament, in: Hans J. Gießmann / Armin Wagner (Hrsg.), Armee im Einsatz. Grundlagen, Strategien und Ergebnisse einer Beteiligung der Bundeswehr, Baden-Baden 2009, S. 148 - 159; Tobias M. Wagner, Parlamentsvorbehalt und Parlamentsbeteiligungsgesetz. Die Beteiligung des Bundestages bei Auslandseinsätzen der Bundeswehr, Berlin 2010; Johannes Hinz, Die Rolle der Parlamente beim Auslandseinsatz deutscher und britischer Streitkräfte, in: ZParl, 42. Jg. (2011), H. 2, S. 286 - 306.

16 Vgl. Sandra Dieterich / Hartwig Hummel / Stefan Marschall, Parliamentary War Powers in Europe, a.a.O. (Fn. 6), S. $22 \mathrm{ff}$.

17 BVerfGE 90, S. 286; für das ParlBG siehe BGBl. I 2005, S. 775 sowie für eine kritische Auseinandersetzung mit diesem: Markus Rau, Auslandseinsatz der Bundeswehr: Was bringt das Parlamentsbeteiligungsgesetz?, in: Archiv des Völkerrechts, 44. Jg. (2006), H. 1, S. 93 - 113; Philipp Scherrer, Das Parlament und sein Heer. Das Parlamentsbeteiligungsgesetz, Berlin 2010.

18 Vgl. René Lüddecke, Parlamentarisierung der nationalen Außenpolitik, Baden-Baden 2010, S. 324.

19 Vgl. Rafael Biermann, a.a.O. (Fn. 15); Andreas L. Paulus, a.a.O. (Fn. 15); Dieter Wiefelspütz, a.a.O. (Fn. 15).

20 Manfred Schwarzmeier, Parlamentarische Mitsteuerung. Strukturen und Prozesse informalen Einflusses im Deutschen Bundestag, Wiesbaden 2001, S. 383, S. 385. 
zusammenhangs zwischen der Regierung und der sie tragenden Mehrheitsfraktionen verfügen insbesondere letztere - die Oppositionsfraktionen hingegen nur zu einem deutlich geringeren Grad - über derlei Möglichkeiten. ${ }^{21}$ So betont beispielsweise Rafael Biermann die Bedeutung von „Koalitionsrunden, Fraktionsvorständen, Fraktionsplenen und Arbeitskreisen" 22 , in denen Entscheidungen vorgeprägt würden. Auch in den für diesen Beitrag geführten Interviews wurde auf informelle Einflussmechanismen hingewiesen. ${ }^{23}$ Angeführt wurden beispielsweise die Ansprache von Ministern der eigenen Partei im Rahmen von Fraktionssitzungen und Sitzungen von Fachausschüssen der Fraktion oder in „Frühstücksrunden“ zwischen Ministern und hochrangigen Abgeordneten (Ausschussvorsitzende, Sprecher etc.). Weiterhin benannt wurden parlamentarische Anträge sowie der Weg in beziehungsweise über die Öffentlichkeit. Die informelleren Möglichkeiten gewinnen zusätzlich an Gewicht, wenn sie mit den formellen Kompetenzen (wie etwa dem ParlBG) gekoppelt werden können. Vor diesem Hintergrund verweist René Lüddecke auf „eine Kultur der vorauseilenden Information des Parlaments“ seitens der Bundesregierungen, um „formellen Kontrollaktivitäten zuvorzukommen“ oder um die Mehrheitsfähigkeit von Positionen zu testen. ${ }^{24}$

Die auf den ersten Blick starke Stellung des Bundestags erfährt jedoch zwei Einschränkungen. Eine resultiert aus den Kompetenzen der Bundesregierung. Andreas L. Paulus spricht in diesem Zusammenhang von „strukturellen Vorteile[n] der Exekutive“25. Diese zeigen sich darin, dass der Anstoß zur Beteiligung der Bundeswehr an einer multinationalen Mission stets von einem Beschluss des Kabinetts ausgeht. Der Bundestag verfügt nicht über ein Initiativrecht. Darüber hinaus werden im Regierungsbeschluss alle maßgeblichen inhaltlichen Parameter eines Einsatzes festgelegt. Dies betrifft beispielsweise die Höchstzahl der einzusetzenden Kräfte, deren Aufgaben und Einsatzgebiet, die Kosten des Einsatzes sowie die Einsatzdauer etc. ( $\$ 3$ ParlBG). Inhaltliche Änderungen dieser Vorgaben durch den Bundestag sind nicht möglich. Seine Entscheidungsrechte beziehen sich demnach ausschließlich darauf, dem von der Bundesregierung vorgelegten Antrag im Ganzen zuzustimmen oder ihn abzulehnen.

Hinzu kommt, dass die Bundesregierung gegenüber dem Bundestag internationale „Vorfestlegungen“ geltend machen kann, von denen sie sich aus Gründen wie Bündnissolidarität oder außenpolitischer Berechenbarkeit nicht lösen kann (oder will). Entsprechend schwerer sollte es dem Bundestag fallen, einen Einsatz der Bundeswehr, den die Bundesregierung etwa bereits im Rahmen von EU oder NATO abgesprochen hat, abzulehnen.

21 Siehe ebenda für eine ausführliche Erörterung der Möglichkeiten der Regierungsfraktionen, auf die Bundesregierung Einfluss zu nehmen.

22 Rafael Biermann, a.a.O. (Fn. 15), S. 617.

23 Interview, MdB, Mitglied des Auswärtigen Ausschusses, FDP-Fraktion, 4. April 2011; Interview, MdB, Mitglied der SPD-Fraktionsführung, 7. April 2011; Interview, MdB, Mitglied des Auswärtigen Ausschusses, SPD-Fraktion, 18. Juli 2011; Interview, MdB, Mitglied des Verteidigungsausschusses, Fraktion Bündnis 90/Die Grünen, 15. April 2011; Interview, ehemaliger Referent für Sicherheitspolitik, CDU/CSU-Fraktion, 2. März 2011.

24 René Lüddecke, a.a.O. (Fn. 18), S. 315.

25 Andreas L. Paulus, a.a.O. (Fn. 15), S. 103. 


\section{Begrenzungen der Einflussmöglichkeiten des Bundestags}

Die Kompetenzen der Bundesregierung sind jedoch nicht der einzige Grund, weshalb die „war powers“ des Bundestags begrenzt sind. Weitere Einschränkungen beziehen sich auf eine geringe Konfliktbereitschaft der Regierungsfraktionen, auf die Uneinigkeit innerhalb der Opposition wie auch auf die Uneinigkeit zwischen Fachpolitikern. Unter Bezugnahme auf die oben angeführte Unterteilung von Born und Hänggi ${ }^{26}$ besteht bei den Mehrheitsfraktionen das Problem darin, dass ihre Mitglieder zwar eine hohe „authority“ gegenüber der Regierung haben, sie also zur Rechenschaft ziehen können. Gleichzeitig verfügen sie allerdings über eine geringe ,attitude“, ihre Kompetenzen auch einzusetzen. Den Oppositionsfraktionen fehlt hingegen „authority“. Obendrein schmälert die fehlende einheitliche Haltung gegenüber der Regierung auch noch die „attitude“, die Regierung zu beeinflussen beziehungsweise zur Rechenschaft zu ziehen. Bei den Fachpolitikern ist zwar die "ability“ hoch, denn bei ihnen sind Ressourcen und Expertise vorhanden; ihnen mangelt es allerdings sowohl an „authority“ (vor allem wenn sie aus den Reihen der Opposition kommen) als auch an „attitude“ (vor allem wenn sie aus den regierungstragenden Fraktionen kommen). Die uneinheitliche Haltung unter den Fachpolitikern erzeugt keinen nennenswerten Druck auf die Bundesregierung, bei ihren Überlegungen zur Formulierung der Mandatstexte auf die Forderungen der Parlamentarier einzugehen. Die folgenden Ausführungen verdeutlichen diese Einordnungen.

\subsection{Geringe Konfliktbereitschaft der Regierungsfraktionen in der Öffentlichkeit}

Eine Begrenzung der Einflussmöglichkeiten des Bundestags ergibt sich aus Zwängen, die parlamentarischen Regierungssystemen zueigen sind und dort auf die Regierungsfraktionen einwirken. Im Unterschied zu präsidentiellen Regierungssystemen gehen in parlamentarischen Systemen Regierungen aus der Mehrheit der Mitglieder des Parlaments hervor und werden von dieser anschließend getragen. Folglich stehen sich in parlamentarischen Systemen nicht die Regierung und das Parlament gegenüber. Vielmehr ergibt sich ein „Dualismus“27 zwischen Parlamentsmehrheit (Regierungsfraktionen) und Regierung auf der einen und parlamentarischer Opposition auf der anderen Seite. Für das Parlament bedeutet dies, dass für die Regierungsfraktionen die Entscheidungsfindung (vor allem in der Gesetzgebung) maßgeblich ist, während sich die Opposition auf die öffentlichkeitswirksame Kontrolle der Regierung und die Artikulation ihrer Positionen konzentriert beziehungsweise aufgrund fehlender Mehrheit konzentrieren muss. ${ }^{28}$ Die sich hieraus ergebenden unterschiedlichen Interessenlagen machen die Einnahme einer einheitlichen Position durch den Bundestag, schon gar gegenüber der Regierung, zur strukturfremden Ausnahme.

26 Vgl. Hans Born / Heiner Hänggi, a.a.O. (Fn. 14).

27 Winfried Steffani, a.a.O. (Fn. 4), S. 155. Später wurde dieser als „neuer“ Dualismus bezeichnet, der den „alten“, das Gegenüber von Parlament als Ganzem und Regierung, abgelöst, mindestens überlagert habe.

28 Manfred Schwarzmeier, a.a.O. (Fn. 20), S. 129 spricht von „primären Mitsteuerungsfunktionen“ der Regierungsfraktionen und „sekundären Mitsteuerungsfunktionen“ der Oppositionsfraktionen. 
In parlamentarischen Regierungssystemen hängt das „Schicksal“ der Regierung von der Zustimmung der sie tragenden Fraktionen ab. Letztere verfügen entsprechend über ein hohes Maß an „authority“. Zugleich ist anzuführen, dass die für Entsendeentscheidungen zu konstatierende ex ante-Vetomacht des Bundestags ein Recht ist, das faktisch ausschließlich die Regierungsfraktionen - aufgrund ihrer Mehrheit - nutzen können. ${ }^{29}$ Die Wahrscheinlichkeit, dass sie von diesem Recht Gebrauch machen, ist allerdings gering. Die Logik des Parlamentarismus und seines neuen Dualismus' besteht eben darin, dass die Parlamentsmehrheit bei Abstimmungen „ihre“ Regierung unterstützt. Dies ist nicht das Ergebnis hierarchischer Zwangsmaßnahmen oder gar Sanktionsdrohungen, sondern dokumentiert das Funktionieren der systemspezifischen Handlungseinheit Regierungsmehrheit. Am Ende eines komplexen Zusammenspiels zwischen Regierungsmitgliedern, Fraktionsführungen, Fraktionsexperten und Gesamtfraktion bei der Willensbildung, die der gegenseitigen Abhängigkeit Rechnung trägt, steht eine - nötigenfalls mehrheitlich - getroffene Entscheidung, die von allen nach außen vertreten wird, auch wenn am Anfang unterschiedliche Positionen existierten. Der Preis, diese gegen den Willen der eigenen Regierung durchzusetzen, käme einem „Vertrauensentzug “30 gleich. Die politischen Folgen könnten bis zur „Auflösung der Regierungskoalition und Neuwahlen“31 reichen sowie international die Verlässlichkeit Deutschlands als Bündnispartner in Frage stellen. Den Regierungsfraktionen fehlt demnach „attitude“. Entsprechend gering schätzen Dieterich, Hummel und Marschall die Wahrscheinlichkeit ein, dass die Mehrheitsfraktionen die ihnen zukommende Möglichkeit zur Abwahl der Regierung nutzen. ${ }^{32}$

Biermann spricht von „einem starken Zustimmungsdruck“33, der auf den Regierungsfraktionen liege. Schwarzmeier verweist hinsichtlich der Verhaltenserwartungen an Mitglieder der Mehrheitsfraktionen auf eine „Metanorm“, die laute, mit dem eigenen Verhalten der Regierung und den sie tragenden Fraktionen „keinen Schaden zuzufügen“ 34 . Und Lüddecke konstatiert, dass sich „die Mitglieder der jeweiligen Regierungsfraktionen aufgrund ihres kollektiven Rollenverständnisses eher als Träger und nicht als formeller Kontrolleur der Regierung sehen " 35 . Eine bestenfalls geringe öffentlich ausgetragene Konfliktbereitschaft der Regierungsfraktionen gegenüber der Regierung ist die naheliegende Folge. Die Konfliktbereitschaft der Abgeordneten stößt spätestens dann an ihre Grenzen, wenn Mehrheiten bei Abstimmungen, und damit möglicherweise der Fortbestand der Regierung, gefährdet sind. Diese Perspektive sollte andere mögliche Einflussfaktoren auf die Haltung der Abgeordneten, wie etwa die Bedeutung eines Einsatzes, überlagern.

29 Vgl. Dirk Peters / Wolfgang Wagner / Cosima Glahn, Parliamentary Control of Military Missions. The Case of the EU NAVFOR Atalanta, RECON Online Working Paper 24, Oslo 2011, S. 11.

30 Rafael Biermann, a.a.O. (Fn. 15), S. 618.

31 Interview, MdB, Mitglied des Verteidigungsausschusses, SPD-Fraktion, 5. April 2011.

32 Vgl. Sandra Dieterich / Hartwig Hummel / Stefan Marschall, Von der exekutiven Prärogative zum parlamentarischen Frieden? Funktionslogik und Funktionsprobleme der parlamentarischen Kontrolle militärischer Sicherheitspolitik am Fallbeispiel Deutschland, PAKS Working Paper 6, Düsseldorf 2007, S. 14.

33 Rafael Biermann, a.a.O. (Fn. 15), S. 618.

34 Manfred Schwarzmeier, a.a.O. (Fn. 20), S. 383.

35 René Lüddecke, a.a.O. (Fn. 18), S. 312. 
Angesichts dieser Zwänge kann es nicht überraschen, dass der Bundestag bislang keinen einzigen Antrag der Bundesregierung zur Entsendung der Bundeswehr abgelehnt hat. ${ }^{36}$ Am nächsten kamen noch die Abstimmung zur Entsendung deutscher Streitkräfte in die NATO-geführte Mission „Essential Harvest“37 in der ehemaligen jugoslawischen Republik Mazedonien im August 2001 sowie die Abstimmung zur Beteiligung an der Antiterrormission „Operation Enduring Freedom“ $(\mathrm{OEF})^{38}$ wenige Monate später. Infolge der Ablehnung des Einsatzes durch mehr als 20 Abgeordnete aus den Fraktionen von SPD und Bündnis 90/Die Grünen fand der Antrag der rot-grünen Regierung unter Bundeskanzler Gerhard Schröder (SPD) zur Beteiligung an "Essential Harvest“ keine eigene Mehrheit. ${ }^{39}$ Aufgrund der - vorher bereits bekannten - Zustimmung von Teilen der Opposition blieben diese Widerstände allerdings ohne Folgen.

Der mit der fehlenden eigenen Mehrheit verbundene Ansehensverlust für die Bundesregierung bei „Essential Harvest“ war jedoch einer der Gründe, warum Schröder wenige Monate später die Abstimmung zu OEF mit der Vertrauensfrage nach Art. 68 GG verband. Durch diese „disziplinierende“ Maßnahme sollte die Kanzlermehrheit für den Regierungsantrag gesichert werden, was auch gelang. ${ }^{40}$ Bezeichnend war in diesem Zusammenhang das Abstimmungsverhalten von Bündnis 90/Die Grünen. Acht Fraktionsmitglieder hatten im Vorfeld der Entscheidung öffentlich dargelegt, weshalb sie gegen eine deutsche Beteiligung stimmen wollten. ${ }^{41}$ Um jedoch den Fortbestand der Regierung nicht zu gefährden, votierten vier der acht Abgeordneten - nach vorheriger Absprache - schließlich mit "Ja“. Der Widerstand der Parlamentarier endete somit an dem Punkt, an dem der Fortbestand der Regierung auf dem Spiel stand. Ein damaliges Mitglied der Fraktion Bündnis 90/Die Grünen konstatierte, dass man sich „eine uneinheitliche Fraktionshaltung [...] nur in der Opposition leisten “42 könne. Die OEF-Entscheidung verdeutlicht die auf Mehrheitsfraktionen lastenden Zwänge, einerseits die eigene Regierung, deren Fortbestand als gemein-

36 Auch sein Rückholrecht hat der Bundestag noch nicht genutzt. Der bislang einzige diesbezügliche Antrag - die Fraktion Die Linke forderte Ende Oktober 2011 den Abzug der Bundeswehr aus Afghanistan - fand keine Mehrheit im Bundestag. Vgl. Deutscher Bundestag, Antrag der Abgeordneten Wolfgang Gehrcke et al. und der Fraktion Die Linke, Drs. 17/7547 vom 26. Oktober, Berlin 2011.

37 Das Ziel dieser in der zweiten Jahreshälfte 2001 durchgeführten Mission bestand darin, zur Verhinderung eines drohenden Bürgerkriegs in Mazedonien vor Ort Waffen von bewaffneten Gruppen einzusammeln und unschädlich zu machen.

38 OEF wurde infolge der Anschläge des 11. September 2001 etabliert und hatte unter anderem zum Ziel, Terroristen zu bekämpfen und deren Einrichtungen (zum Beispiel Trainingscamps) zu zerstören. Deutschland nahm zwischen 2001 und 2010 an OEF teil. Geographisch lag der Einsatzschwerpunkt der Bundeswehr in Afghanistan und am Horn von Afrika.

39 Vgl. Rafael Biermann, a.a.O. (Fn. 15), S. 616.

40 Vgl. Franz-Josef Meiers, Der wehrverfassungsrechtliche Parlamentsvorbehalt und die Verteidigung der Sicherheit Deutschlands am Hindukusch, 2001-2011, in: Klaus Brummer / Stefan Fröhlich (Hrsg.), Zehn Jahre Deutschland in Afghanistan, Wiesbaden 2011, S. 87 - 113, S. 90 ff.

41 Vgl. Annelie Buntenbach / Steff Lemke / Christian Simmert / Winfried Hermann / Monika Knoche / Irmingard Schewe-Gerigk / Hans-Christian Ströbele / Sylvia Voß, Krieg in Afghanistan. Positionspapier vom 10. November 2001, http://www.stroebele-online.de/show/3792.html?searchshow= positionspapier (Abruf am 26. April 2011).

42 Interview, ehemaliges MdB und Mitglied des Verteidigungsausschusses, Fraktion Bündnis 90/Die Grünen, 14. April 2011. 
sames Ziel außer Frage steht, zu unterstützen, andererseits im Einzelfall abweichende Positionen öffentlich hinreichend zur Geltung zu bringen.

\subsection{Uneinigkeit innerhalb der Opposition}

Eine weitere Begrenzung der „war powers“ des Bundestags resultiert aus der Uneinigkeit innerhalb der Opposition in diesbezüglichen Fragen. Im Unterschied zu den Regierungsfraktionen, die stets weitestgehend geschlossen hinter ihrer Regierung stehen, zeigen die Oppositionsfraktionen kein einheitliches Abstimmungsverhalten. Das über die letzten beiden Jahrzehnte deutlich gewordene „Oppositionsmuster“ sieht die CDU/CSU-Fraktion, das den Regierungsanträgen mit großer Mehrheit folgt, an einem Ende des Spektrums und die frühere PDS-Fraktion beziehungsweise die heutige Fraktion Die Linke, die stets gegen Auslandseinsätze stimmt, am anderen Ende. Dazwischen liegen die Fraktionen von SPD, Bündnis 90/Die Grünen und FDP, die sich einmal für und einmal gegen eine deutsche Beteiligung an einer multinationalen Mission aussprechen.

Die CDU/CSU-Fraktion stimmte während der rot-grünen Regierung (1998 bis 2005) in der Regel mit großen Mehrheiten für die Anträge der Regierung Schröder. Neben der inhaltlichen Unterstützung der Einsätze gründete die Haltung der Fraktion auf dem Bestreben, den ins Ausland gehenden Soldaten eine möglichst breite parlamentarische Legitimation zu verschaffen und somit Rückhalt zu geben. Die größte Ausnahme war die Ablehnung des OEF-Mandats Ende 2001. Dies lag jedoch nicht an inhaltlichen Bedenken - die Unionsfraktion hatte im Vorfeld der Plenarentscheidung ihre Unterstützung signalisiert -, sondern an der geschilderten Verknüpfung der Abstimmung zu OEF mit der Vertrauensfrage. ${ }^{43}$

Die Fraktion Die Linke, die Auslandseinsätze der Bundeswehr kategorisch ablehnt und sich entsprechend konsequent gegen diesbezügliche Regierungsanträge stellt, zeigte eine der „größten Abweichungen“ von dieser Regel im Falle eines VN-geführten Einsatzes im sudanesischen Darfur (UNAMID) im November 2007. Während 38 Fraktionsmitglieder gegen den Regierungsantrag stimmten, enthielten sich acht. ${ }^{44}$ Diese Uneinigkeit war umso bemerkenswerter, weil mehrere Spitzenvertreter der Fraktion zu den „Abweichlern“ zählten: Mit Gregor Gysi, Bodo Ramelow und Gesine Lötzsch enthielten sich einer der beiden Fraktionsvorsitzenden und zwei stellvertretende Fraktionsvorsitzende. Die fehlende Geschlossenheit bei UNAMID stellt jedoch die Ausnahme zum ansonsten üblichen, einvernehmlich ablehnenden Abstimmungsverhalten der Linken dar.

Die Zustimmung beziehungsweise Ablehnung der Fraktionen von SPD, Bündnis 90/ Die Grünen und FDP ist abhängig vom konkreten Einsatz. Deutlich wird die - je nach Sichtweise positiv einzuschätzende Offenheit oder aber negativ zu bewertende fehlende Kohärenz - am Abstimmungsverhalten der FDP-Fraktion während der Großen Koalition. Einerseits unterstützten die Freidemokraten beispielsweise mehrmals nahezu geschlossen die Verlängerung des ISAF-Einsatzes in Afghanistan, und im Dezember 2008 stimmten sogar alle Fraktionsmitglieder für die Beteiligung an der EU-geführten Antipirateriemission

43 Vgl. Klaus Brummer / Stefan Fröhlich, Einleitung: Zehn Jahre Deutschland in Afghanistan, in: dies. (Hrsg.), Zehn Jahre Deutschland in Afghanistan, Wiesbaden 2011, S. 3 - 30, S. 9.

44 Vgl. Deutscher Bundestag, Stenografischer Bericht, Plenarprotokoll 16/126 vom 15. November, Berlin 2007, S. 13147. 
„Atalanta“ vor der somalischen Küste. ${ }^{45}$ Andererseits stellte sich die Fraktion fast ebenso einmütig gegen die Teilnahme der Bundeswehr an der EU-geführten Mission EUFOR RD in der Demokratischen Republik Kongo ${ }^{46}$ oder an UNIFIL (siehe unten).

Ähnlich verhielt sich jüngst die SPD-Fraktion unter der christlich-liberalen Regierungskoalition. Während sie beispielsweise mehrfach das ISAF-Mandat mit großer Mehrheit unterstützte, stellte sie sich gegen die deutsche Beteiligung an OEF wie auch an „Operation Active Endeavour“ (OAE). ${ }^{47}$ Auffällig ist dabei, dass sich die Sozialdemokraten im Falle der Ablehnung eines Einsatzes gleich weitgehend geschlossen gegen diesen wendeten. Bei der Abstimmung zur Verlängerung der Beteiligung an OAE im Dezember 2011 stimmten nur zwei Abgeordnete für den Regierungsantrag. ${ }^{48}$ Bei der vorhergehenden Verlängerung im Dezember 2010 stimmte kein einziges Mitglied der SPD-Fraktion zu. ${ }^{49}$ Selbiges galt für den Antrag zur Verlängerung der Beteiligung an OEF im Dezember 2009.50

Bei Bündnis 90/Die Grünen ist hingegen zu bemerken, dass die Zustimmung beziehungsweise Ablehnung zu Einsätzen wiederholt nicht derart eindeutig ausgefallen ist wie bei der FDP oder der SPD. Ein differenziertes Meinungsbild innerhalb der Grünen-Fraktion zeigte sich beispielsweise im Dezember 2008 bei der Abstimmung zu Atalanta, als 35 Mitglieder der Fraktion mit "Ja“ stimmten, sechs mit „Nein“ und acht sich enthielten. 51 Bei der Verlängerung des ISAF-Einsatzes im Januar 2012 stimmten zehn Abgeordnete für den Regierungsantrag, 19 dagegen und 30 enthielten sich. ${ }^{52}$ Ähnlich uneinheitlich verhielt sich die Fraktion bei der noch zu diskutierenden Entscheidung zur Beteiligung an UNIFIL.

Kurzum: Die Oppositionsfraktionen zeigen ein wenig kohärentes Abstimmungsverhalten. Zwar besteht die Aufgabe der Opposition in pluralistischen Demokratien gerade darin, vielfältige - und sich somit voneinander unterscheidende - Alternativen zur Regierungsposition anzubieten. Vor dem Hintergrund des angeführten Dualismus zwischen Regierung und Parlamentsmehrheit auf der einen, Opposition auf der anderen Seite führt eine uneinheitliche Haltung letzterer jedoch unweigerlich zu deren Schwächung gegenüber der Bundesregierung. Von der Opposition kann in diesen Fällen kein nennenswerter Druck ausgehen. Neben der „authority“ gegenüber der Bundesregierung mangelt es somit auch an „attitude“, diese zur Rechenschaft zu ziehen. Ein Mitglied des Verteidigungsausschusses sprach sogar von der „Ohnmacht der Opposition“53.

45 Vgl. Deutscher Bundestag, Stenografischer Bericht, Plenarprotokoll 16/197 vom 19. Dezember, Berlin 2008, S. 21359.

46 Vgl. Deutscher Bundestag, Stenografischer Bericht, Plenarprotokoll 16/37 vom 1. Juni, Berlin 2006, S. 3261.

47 OAE wurde als Reaktion auf die Anschläge des 11. September 2001 etabliert. Die im Mittelmeer durchgeführte Mission dient vor allem der Terrorismusbekämpfung.

48 Vgl. Deutscher Bundestag, Stenografischer Bericht, Plenarprotokoll 17/146 vom 1. Dezember, Berlin 2011, S. 17388.

49 Vgl. Deutscher Bundestag, Stenografischer Bericht, Plenarprotokoll 17/78 vom 2. Dezember, Berlin 2010, S. 8650.

50 Vgl. Deutscher Bundestag, Stenografischer Bericht, Plenarprotokoll 17/9 vom 3. Dezember, Berlin 2009, S. 712.

51 Vgl. Deutscher Bundestag, a.a.O. (Fn. 45), S. 21359 f.

52 Vgl. Deutscher Bundestag, Stenografischer Bericht, Plenarprotokoll 17/155 vom 26. Januar, Berlin 2012, S. $18577 \mathrm{f}$.

53 Interview, MdB, Mitglied des Verteidigungsausschusses, Fraktion Bündnis 90/Die Grünen, 15. April 2011. 
Für die Bundesregierung wiederum ergibt sich aus der fehlenden Geschlossenheit der Opposition keine zwingende Notwendigkeit, bei ihren Beratungen über Mandatstexte sowie abschließend in ihrem Antrag an den Bundestag auf deren Forderungen einzugehen. Vielmehr kann eine Bundesregierung gleich welcher „Couleur“ davon ausgehen, dass zumindest eine der Oppositionsfraktionen mehrheitlich ihren Anträgen folgen wird. In den meisten Fällen findet sich somit - wie von der Bundesregierung gewünscht ${ }^{54}$ - eine auch Teile der Opposition einbeziehende breite Mehrheit im Bundestag für Entsende-Entscheidungen. Durch die Einbindung von Oppositionsfraktionen kann die Bundesregierung „das

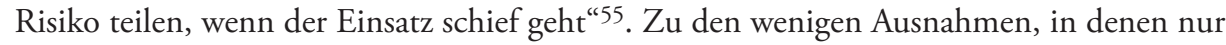
die Regierungsfraktionen für einen Antrag stimmten, gehörten die Entscheidungen zur Teilnahme an OEF im November 2001 und zur Verlängerung von OAF im Dezember 2010 .

\subsection{Uneinigkeit unter Fachpolitikern}

Ein dritter Faktor, der die Einflussmöglichkeiten des Bundestags begrenzt, bezieht sich auf die fraktionsübergreifende Uneinigkeit unter Fachpolitikern. Mit Blick auf die Auslandseinsätze der Bundeswehr gibt es diese insbesondere zwischen dem Auswärtigen Ausschuss und dem Verteidigungsausschuss beziehungsweise zwischen „Außenpolitikern“ und „Verteidigungspolitikern“. Der Auswärtige Ausschuss und der Verteidigungsausschuss sind die beiden maßgeblichen Gremien des Bundestags mit Blick auf die parlamentarische Befassung mit Auslandseinsätzen der Bundeswehr. Federführend ist dabei der Auswärtige Ausschuss, den Volker Pilz generell als „das zentrale Organe zur außenpolitischen Willensbildung des Parlaments"56 bezeichnet. Der Verteidigungsausschuss gehört zu den mitberatenden Ausschüssen, wobei er schon allein aufgrund der inhaltlichen Nähe zur Thematik unter diesen hervorsticht. ${ }^{57}$ Die besondere Stellung beider Gremien zeigt sich auch daran, dass es zu gemeinsamen Sitzungen zu Fragen der Auslandseinsätze kommt. Dies war beispielsweise im März 2006 der Fall, als die Bundesregierung den Auswärtigen Ausschuss und den Verteidigungsausschuss in einer gemeinsamen Sitzung über den Stand der Planungen zu EUFOR RD Congo informierte. ${ }^{58}$

Trotz dieser Nähe verweist Pilz auf unterschiedliche Zugänge zur Thematik. ${ }^{59}$ Während sich der Auswärtige Ausschuss vor allem mit dem „Ob“ eines Einsatzes befasse, beschäftige

54 Vgl. Andreas L. Paulus, a.a.O. (Fn. 15), S. 91.

55 Interview, MdB, Mitglied der SPD-Fraktionsführung, 7. April 2011.

56 Volker Pilz, Der Auswärtige Ausschuss des Deutschen Bundestages und die Mitwirkung des Parlaments an der auswärtigen und internationalen Politik, Berlin 2008, S. 123 (Hervorhebungen entfernt).

57 Beteiligt sind in der Regel auch der Rechtsausschuss, der Haushaltsausschuss, der Ausschuss für Menschenrechte und Humanitäre Hilfe, der Ausschuss für wirtschaftliche Zusammenarbeit und Entwicklung und der Ausschuss für die Angelegenheiten der Europäischen Union. Bei den letzten drei Ausschüssen hängt ihre Einbindung vom konkreten Einsatz (Einsatzgebiet, durchführende Organisation etc.) ab.

$58 \mathrm{Vgl}$. Jan Ryjáček, Der Entscheidungsprozess über den Bundeswehreinsatz zum Schutz der Wahlen im Kongo, a.a.O. (Fn. 15), S. 227.

59 Vgl. Volker Pilz, a.a.O. (Fn. 56), S. 70. 
sich der Verteidigungsausschuss vorwiegend mit dem „Wie“. Aufgrund dieser unterschiedlichen Sichtweisen komme es mitunter zu unterschiedlichen Einschätzungen. Uneinigkeit zwischen den Fachpolitikern kann dabei ganz grundsätzlich über die Notwendigkeit eines Einsatzes bestehen. ${ }^{60}$ Überraschend ist in diesem Zusammenhang, dass Außenpolitiker Auslandseinsätzen „offener"61 gegenüberzustehen scheinen als Verteidigungspolitiker. Darüber hinaus können sich Unstimmigkeiten zwischen den Fachpolitikern auch auf die operative Ausgestaltung eines Einsatzes beziehen. In diesem Sinne bemerkte ein Mitglied des Verteidigungsausschusses, dass es eben „oft verschiedene Möglichkeiten für Einsätze“62 gebe.

Die von Jan Ryjáček konstatierte „Rivalität zwischen dem Auswärtigen und dem Verteidigungsausschuss in der Frage der Auslandseinsätze" 63 scheint somit zuzutreffen. Sie wurde auch von Abgeordneten des Bundestags bestätigt. Ein Mitglied des Auswärtigen Ausschusses verwies auf den von Verteidigungspolitikern gegen seinen Ausschuss gemachten Vorwurf, ein „Wolkenschieberausschuss " ${ }^{" 64}$ zu sein, der sich in abstrakten Diskussionen ergehe. Es hob stattdessen die „gute Diskussionskultur“ sowie die Möglichkeit zu offenen Diskussionen ,jenseits des Parteibuchs“ als zentrale Eigenschaften hervor. ${ }^{65}$ Ein Mitglied des Verteidigungsausschusses gestand dem Auswärtigen Ausschuss wiederum durchaus Expertise zu und unterstützte auch dessen federführende Rolle bei Auslandseinsätzen angesichts des „Primats der Politik“66. Dennoch ließ der Befragte keinen Zweifel daran, dass der Verteidigungsausschuss „zentral“67 sei, da er sich wesentlich häufiger mit der Materie befasse. Und ein anderer Verteidigungspolitiker sprach davon, dass seinem Ausschuss die „faktische Federführung "68 bei Auslandseinsätzen zukomme.

Pilz betont sicherlich zu Recht, dass sich die Bundesregierung ,in aller Regel kaum entgegen dem Willen der sie tragenden Mehrheit verhalten "69 wird. Aus der obigen Diskussion ist dem allerdings entgegenzuhalten, dass dieser „Wille“ („attitude“) oftmals nicht erkennbar ist aufgrund unterschiedlicher inhaltlicher Positionierungen der Fraktionen wie auch der Fachpolitiker innerhalb der (wie auch zwischen den) Fraktionen. Wie die Literatur zu „epistemic communities“ zeigt, beruht der Einfluss von Experten jedoch nicht nur auf deren Fachwissen, sondern auch auf ihrer weitgehend geschlossenen Haltung zu einer

60 Die Unterschiede reichen allerdings nicht soweit, dass ein Einsatz in einem Ausschuss befürwortet und in dem anderen Ausschuss abgelehnt würde. Eine Ablehnung dürfte schon durch die weiter oben angeführte Mehrheit der Regierungsfraktionen in den Ausschüssen verhindert werden. Jedenfalls ist dem Verfasser kein Fall bekannt, in dem einer der beiden Ausschüsse mehrheitlich gegen einen Antrag der Regierung gestimmt hätte.

61 Interview, ehemaliges MdB und Mitglied des Verteidigungsausschusses, Fraktion Bündnis 90/Die Grünen, 14. April 2011; Interview, ehemaliger Referent für Sicherheitspolitik, CDU/CSU-Fraktion, 2. März 2011.

62 Interview, MdB, Mitglied des Verteidigungsausschusses, SPD-Fraktion, 5. April 2011.

63 Jan Ryjáček, Der Entscheidungsprozess über den Bundeswehreinsatz zum Schutz der Wahlen im Kongo, a.a.O. (Fn. 15), S. 232.

64 Interview, MdB, Mitglied des Auswärtigen Ausschusses, FDP-Fraktion, 4. April 2011.

65 Ebenda.

66 Interview, MdB, Mitglied des Verteidigungsausschusses, SPD-Fraktion, 5. April 2011.

67 Ebenda.

68 Interview, ehemaliges MdB und Mitglied der SPD-Fraktionsführung, 13. April 2011.

69 Volker Pilz, a.a.O. (Fn. 56), S. 121. 
Sachfrage. ${ }^{70}$ Da es unter den Fachpolitikern des Bundestags an Letzterem zu mangeln scheint, verliert ihre Expertise („ability“) an Einfluss, zumal wenn die Fachpolitiker aus den Reihen der Opposition kommen, was zusätzlich ihre „authority“ schmälert. Dass die drei diskutierten Handlungsbegrenzungen auch in der politischen Praxis vorkommen, illustriert der folgende Abschnitt.

\section{United Nations Interim Force in Lebanon $(\text { UNIFIL) })^{71}$}

\subsection{Fallillustration}

Den Hintergrund für die deutsche Beteiligung an der „United Nations Interim Force in Lebanon“ (UNIFIL) bildete die Eskalation des Konflikts zwischen Israel und der Hisbollah-Miliz im Juli 2006. Als Reaktion auf Raketenangriffe sowie Entführungen israelischer Soldaten durch die Hisbollah überquerte die israelische Armee am 19. Juli die Grenze zum Libanon. Daraufhin erweiterte der VN-Sicherheitsrat Mitte August in seiner Resolution 1701 das Mandat wie auch die Personalstärke der bereits seit 1978 laufenden UNIFILMission. ${ }^{72}$ Die Truppenstärke sollte von etwa 2.000 auf bis zu 15.000 Soldaten angehoben werden. Die Mission sollte fortan unter anderem die Beendigung der Feindseligkeiten überwachen und sicherstellen, dass humanitäre Helfer Zugang zur Zivilbevölkerung erhielten. Eine weitere Neuerung bestand darin, dass UNIFIL auf Ersuchen der libanesischen Regierung dieser dabei helfen sollte, die Grenzen des Landes zu sichern, um den Schmuggel von Rüstungsgütern zu verhindern.

Nach der Verabschiedung der VN-Resolution und der Einleitung eines Waffenstillstands am 14. August stand die Konkretisierung des Einsatzes im Mittelpunkt der internationalen Bemühungen. Der Durchbruch erfolgte Ende August, als mehrere Mitgliedstaaten der EU bis zu 6.900 Soldaten für UNIFIL zusagten. Anfang September traf das erste größere, vor allem von italienischen Soldaten gebildete Kontingent der reformierten UNIFIL-Truppe im Libanon ein. Die neue maritime Komponente der Mission lief ebenfalls im September an.

Am 13. September verabschiedete die Bundesregierung ihren Antrag an den Bundestag zur Beteiligung der deutschen Marine an UNIFIL. ${ }^{73}$ Darin war eine Beteiligung von bis zu 2.400 Soldatinnen und Soldaten vorgesehen, insbesondere aus den Reihen der Marine. Der deutsche Beitrag sollte entsprechend vorrangig „seegestützt sein und militärische Fähigkeiten zur UNIFIL-Überwachung des libanesischen Küstenbereiches umfassen "74. Der Einsatz, dessen Kosten auf 193 Millionen Euro beziffert wurden, war zunächst bis Ende

70 Vgl. Ernst B. Haas, When Knowledge is Power. Three Models of Change in International Organizations, Berkeley / Los Angeles / Oxford 1990.

71 Die Fallauswahl wird im einleitenden Abschnitt dieses Beitrags begründet.

72 UNIFIL war im März 1978 vor dem Hintergrund einer Kommandoaktion der vom Südlibanon aus agierenden Palästinensischen Befreiungsorganisation (PLO), der zahlreiche israelische Zivilisten zum Opfer gefallen waren, und der darauf folgenden Invasion Südlibanons durch Israel etabliert worden. Bis zum Jahr 2006 war die Bundesrepublik nicht an UNIFIL beteiligt.

73 Vgl. Deutscher Bundestag, Antrag der Bundesregierung, Drs. 16/2572 vom 13. September, Berlin 2006.

74 Ebenda, S. 3. 


\begin{tabular}{|l|c|c|c|}
\hline Tabelle 1: Bundestagsabstimmung zum UNIFIL-Einsatz am 20. September 2006 \\
\hline Abstimmungsverhalten Fraktionen & „Ja-Stimmen & „Nein“-Stimmen & Enthaltungen \\
\hline CDU/CSU & 208 & 12 & 1 \\
SPD & 186 & 32 & 0 \\
FDP & 8 & 51 & 2 \\
Die Linke & 0 & 50 & 0 \\
Bündnis 90/Die Grünen & 40 & 6 & 2 \\
Fraktionslose & 0 & 1 & 0 \\
\hline Summe & 442 & 152 & 5 \\
\hline Quelle: Vgl. Deutscher Bundestag, Stenografischer Bericht, Plenarprotokoll 16/50 vom 20. September, \\
Berlin 2006, S. 4846.
\end{tabular}

August 2007 befristet. Der Bundestag folgte dem Antrag der Regierung eine Woche später (siehe Tabelle 1). Der deutsche Einsatzverband lief am 21. September aus und übernahm am 15. Oktober die Führung des multinationalen maritimen Einsatzverbandes von UNIFIL. Die Bundeswehr ist bis heute an der Mission beteiligt.

\subsection{Geringe Konfliktbereitschaft der Regierungsfraktionen}

Das Verhalten der Regierungsfraktionen bei der Entscheidung zu UNIFIL weist zwei Charakteristika auf. Erstens gab es sowohl innerhalb der CDU/CSU- als auch innerhalb der SPD-Fraktion kritische Stimmen gegen eine Beteiligung der Bundeswehr. Zweitens spiegelte sich die Kritik nur bedingt im Abstimmungsergebnis wider.

Die Kritik aus den Reihen der Unionsfraktion, und hier insbesondere aus der CSU, bezog sich vor allem auf einen möglichen Kampfeinsatz. So sagte der Vorsitzende der CSULandesgruppe im Bundestag, Peter Ramsauer: „Für mich ist die Zustimmung zu einer Beteiligung deutscher Soldaten an einer kämpfenden Truppe nicht denkbar. "75 Noch Anfang September, als Schwierigkeiten bei der Missionsplanung auftraten, betonte Ramsauer, dass diese gelöst werden müssten. Falls nicht, so Ramsauer weiter, „dann gehen wir eben nicht hin"76. Dennoch gab es letztlich nur ein Dutzend „Nein“-Stimmen aus der Unionsfraktion. Angesichts der Widerstände, die sich nicht nur gegen einen Kampfeinsatz richteten, sondern auch aus historischen Gründen allgemein gegen einen Einsatz der Bundeswehr im Nahen Osten, liegt die Vermutung nahe, dass die Zugehörigkeit zum „Regierungslager“ eine wichtige Rolle für das Hintanstellen der inhaltlichen Bedenken spielte.

Der deutlich größere Teil der „Nein“-Stimmen aus den Regierungsfraktionen stammte aus den Reihen der SPD. Mit 32 Gegenstimmen überraschte das Ausmaß der Ablehnung sogar die Fraktionsführung. Zwei Tage vor der Abstimmung im Bundestag war der SPDFraktionsvorsitzende Peter Struck von nur „einige[n] wenige[n] Gegenstimmen“77 ausge-

75 Ansgar Graw / Mariam Lau, In der Kampfzone, in: Die Welt vom 16. August 2006, S. 3.

76 Hans-Jürgen Leersch, Steinmeier verhandelt in Beirut über Marine-Einsatz, in: Die Welt vom 8. September 2006, S. 5.

77 „Merkel will noch einmal im Bundestag für den Libanon-Einsatz werben“, in: Associated Press vom 18. September 2006. 
gangen. Vorwarnungen hatte es allerdings gegeben. So hatte beispielsweise der stellvertretende Fraktionsvorsitzende, Ludwig Stiegler, nach der Verabschiedung der VN-Resolution 1701 betont, dass „[e]in gutes Drittel der Fraktion“78 Probleme mit einer militärischen Beteiligung Deutschlands an einer Friedenstruppe habe. Neben inhaltlichen Zweifeln kritisierten Vertreter der SPD auch die Informationspolitik der Regierung gegenüber dem Parlament. Die Vorsitzende des Verteidigungsausschusses, Ulrike Merten (SPD), hätte sich beispielsweise gewünscht, dass Verteidigungsminister Franz Josef Jung den Bundestag „etwas zeitnaher "79 unterrichtet hätte, wodurch die Parlamentarier weniger Informationen aus Zeitungen hätten erhalten müssen. Das von Stiegler angeführte „Drittel der Fraktion“ - was mehr als 70 Abgeordnete bedeutet hätte - stellte sich dann jedoch nicht gegen den Antrag der Bundesregierung. Entsprechend wollte Struck die Abstimmung nicht überbewertet sehen. Er sprach vielmehr von einem „Sonderfall, weil viele persönliche Gründe für sich reklamierten "80.

Strucks Aussage zum „Sonderfall“ verdeutlicht noch einmal, dass der „Regelfall“ darin besteht, dass die Abgeordneten der Regierungsfraktionen den Anträgen der Bundesregierung folgen. Auch der Vorsitzende der CDU/CSU-Fraktion, Volker Kauder, brachte dies unmissverständlich zum Ausdruck. Eine Woche vor der Verabschiedung des Regierungsantrags sagte er: „Über diesen Antrag [der Bundesregierung] werden wir dann beraten. Heute, Frau Bundeskanzlerin, kann ich Ihnen eines schon sagen: Wir werden die Details natürlich ganz genau prüfen, aber das Angebot, das Sie und die Bundesregierung gemacht haben, kann unsere grundsätzliche Zustimmung finden. "81 Der Vorsitzende der größten Bundestagsfraktion signalisierte der Bundesregierung somit die weitgehend bedingungslose $\mathrm{Zu}$ stimmung zu ihrem Antrag, und dies zu einem Zeitpunkt, als die Bundesregierung ihre Entscheidung noch gar nicht getroffen hatte. Die von Kauder gemachte Einschränkung dieses „Freibriefs“ dahingehend, dass der Bundestag die Details des vorzulegenden Antrags prüfen werde, war bloße Rhetorik, da das Parlament schlichtweg nicht die Kompetenzen hat, inhaltliche Änderungen an Regierungsanträgen vorzunehmen.

\subsection{Uneinigkeit innerhalb der Opposition}

Bei der Abstimmung zu UNIFIL zeigte sich die Opposition erneut uneins. Während Die Linke geschlossen und die FDP weitgehend geschlossen gegen den Regierungsantrag stimmten, sprach sich eine klare Mehrheit der Grünen für diesen aus (siehe Tabelle 1). Die Linke führte neben ihrer generellen Ablehnung von Auslandseinsätzen noch mehrere missionsspezifische Aspekte zur Begründung ihrer Haltung an. Dies waren die deutsche Geschichte sowie die zu erwartenden Folgen eines deutschen Einsatzes im Nahen Osten. Mit Blick auf die deutsche Geschichte sagte beispielsweise Gregor Gysi: „Nach der millionenfa-

78 Stefan Weißenborn, Koalition debattiert deutschen Libanon-Einsatz, in: DDP vom 14. August 2006.

79 „Entscheidung über Bundeswehr-Einsatz im Libanon verzögert sich“, in: Agence France Presse vom 3. September 2006.

80 Horand Knaup / Konstantin von Hammerstein, Eine gewisse Nervosität, in: Der Spiegel vom 2. Oktober 2006, S. 28 - 32, S. 32.

81 Deutscher Bundestag, Stenografischer Bericht, Plenarprotokoll 16/46 vom 6. September, Berlin 2006, S. 4500. 
chen Ermordung von Juden durch Deutsche dürfen deutsche Soldaten weder zur Kontrolle eines fragilen Waffenstillstands noch zur Friedenserzwingung Israel gegenüberstehen. " ${ }^{82} \mathrm{Er}$ warnte außerdem davor, dass Deutschland im Falle einer Beteiligung an einer VN-Mission „nicht Teil der Lösung, sondern Teil des Konfliktes“83 im Nahen Osten sein würde. Der zweite Fraktionsvorsitzende, Oskar Lafontaine, führte an, dass ein Einsatz der Bundeswehr die Sicherheit Deutschlands gefährden könnte: „Wer sich überall einlässt - und zwar so einlässt wie Sie [die Bundesregierung] hinsichtlich des Libanon -, der erhöht die Gefahr für Terroranschläge in Deutschland und verletzt den Eid, den Sie hier geleistet haben, nämlich Schaden vom deutschen Volk abzuwenden." 84

Die FDP-Fraktion stellte sich ebenfalls gegen den Regierungsantrag. Die Argumente gegen einen Einsatz ähnelten denjenigen der Linken. Mit Blick auf die Lehren, die aus der deutschen Geschichte gezogen werden müssten, sagte der Bundespartei- und Fraktionsvorsitzende der FDP, Guido Westerwelle: „Wenn es eine Region in der Welt gibt, wo deutsche Soldaten nichts zu suchen haben, dann im Nahen Osten an der Grenze zu Israel. "85 Die sicherheitspolitische Sprecherin der FDP-Fraktion, Brigitte Homburger, bezeichnete die Abstimmung innerhalb der Bundesregierung zu UNIFIL als „Desaster" ${ }^{\circ 6}$, das sich in den fortbestehenden Unklarheiten über das deutsche Angebot an die VN widerspiegele.

Im Unterschied zu den beiden anderen Oppositionsfraktionen unterstützten die Grünen den Antrag der Regierung. Spitzenvertreter der Fraktion zeigten sich von Anfang an offen gegenüber einem Einsatz. Sie machten ihre Entscheidung allerdings abhängig von dessen Ausgestaltung. In diesem Zusammenhang wurde auch deutliche Kritik an der Haltung der beiden anderen Oppositionsfraktionen geübt. So sagte einer der beiden Fraktionsvorsitzenden, Fritz Kuhn: „Wir lehnen diesen pauschalen Fundamentalismus à la Westerwelle und Lafontaine $\mathrm{ab}$, sondern sagen, ob man da zustimmt, hängt von den genauen Umständen des Einsatzes ab. “87

Die „abwartend-offene“ Haltung der beiden Fraktionsvorsitzenden war jedoch nicht unumstritten. Einer der stellvertretenden Vorsitzenden, Jürgen Trittin, gab beispielsweise mit Blick auf die deutsche Geschichte zu bedenken: „Stellen wir uns doch mal folgende Situation vor: Israel verstößt gegen ein Waffenstillstandsabkommen. Dann müssten deutsche Soldaten bei einem robusten Mandat auf israelische Soldaten schießen. Das ist völlig undenkbar. "88 Ein anderer stellvertretender Fraktionsvorsitzender, Hans-Christian Ströbele, führte als weiteres Argument gegen einen Einsatz an, dass die VN-Truppen, und somit auch die Bundeswehrsoldaten, bei einem Konflikt mit dem Iran möglicherweise in diesen hineingezogen werden könnten. ${ }^{89}$

82 „Opposition warnt vor Bundeswehreinsatz“, in: Financial Times Deutschland vom 26. Juli 2006, S. 13.

83 „2.400 Soldaten für Libanon-Einsatz“, in: DDP vom 13. September 2006.

84 Deutscher Bundestag, a.a.O. (Fn. 81), S. 4488.

85 „Bundesregierung nimmt sich für Libanon-Entscheidung Zeit“, in: Associated Press vom 14. August 2006.

86 Hans-Jürgen Leersch, Homburger nennt Vorbereitung des Bundeswehr-Einsatzes „Desaster“, in: Die Welt vom 19. August 2006, S. 6.

87 „Kritik an Jung“, in: FAZ vom 28. August 2006, S. 2.

88 Jörg Michel, Jamaika wäre das Ende der Grünen, in: Berliner Zeitung vom 22. Juli 2006, S. 6.

89 Vgl. „Mehrheit der Grünen im Bundestag für Nahost-Einsatz“, in: Associated Press vom 15. September 2006. 
Trotz dieser Kritik stimmte die Fraktion schließlich mit großer Mehrheit dem Regierungsantrag zu. Als die maßgeblichen Gründe nannte Kuhn die sich aus der deutschen Geschichte ergebende Verpflichtung für das Existenzrechts Israels, die Förderung der staatlichen Souveränität des Libanon, die Stärkung Europas und der VN sowie die Steigerung der deutschen Vermittlerrolle im Nahen Osten. Hinzu käme, dass die Einsatzregeln eine Konfrontation deutscher und israelischer Soldaten ausschlössen. ${ }^{90}$

\subsection{Uneinigkeit unter Fachpolitikern}

Bei der Entscheidung zur Beteiligung an UNIFIL standen die Verteidigungspolitiker der beiden Regierungsfraktionen einem Einsatz deutlich kritischer gegenüber als die Außenpolitiker. Sie hinterfragten insbesondere die Fähigkeiten und Ressourcen der Bundeswehr. Für den verteidigungspolitischen Sprecher der CDU/CSU-Fraktion, Bernd Siebert, war es beispielsweise unklar, woher die Mittel für einen Einsatz der Bundeswehr stammen sollten. ${ }^{91}$ Weiterhin brachte Siebert Bedenken hinsichtlich der materiellen Fähigkeiten der Bundeswehr an: „Soldaten, die nicht die notwendige Ausstattung haben, sind für niemanden eine Hilfe. "92 Der stellvertretende Vorsitzende der SPD-Fraktion und frühere Parlamentarische Staatssekretär beim Bundesminister der Verteidigung, Walter Kolbow, verwies auf die Belastungen der Bundeswehr, die aufgrund bereits laufender Einsätze „nicht grenzenlos einsatzfähig "93 sei.

$\mathrm{Zu}$ den weiteren von Verteidigungspolitikern gegen eine Beteiligung an UNIFIL vorgebrachten Bedenken zählten die mit einem Einsatz einhergehenden Risiken sowie die deutsche Geschichte. Der stellvertretende Vorsitzende des Verteidigungsausschusses, Karl Lamers (CDU), betonte: „Dies ist eine der kritischsten und gefährlichsten Regionen in der Welt, und wir müssen uns darüber im Klaren sein, dass das kein Spaziergang ist. "94 Und der verteidigungspolitische Sprecher der SPD-Fraktion, Rainer Arnold, äußerte sich aufgrund der deutschen Geschichte zurückhaltend gegenüber einem Einsatz im Nahen Osten: „Jeder weiß, dass wir eine besondere Verantwortung für Israel haben, aber die Kehrseite der Medaille ist eine besondere Sensibilität. “95

Demgegenüber zeigten sich Außenpolitiker der Regierungsfraktionen offener für einen Bundeswehreinsatz. Sie führten ebenfalls die deutsche Geschichte an, zogen aus dieser jedoch andere Schlüsse als die Verteidigungspolitiker. So sagte der stellvertretende Vorsitzende der CDU/CSU-Fraktion für die Bereiche Außen-, Verteidigungs- und Europapolitik, Andreas Schockenhoff: „Wenn eine Überwachung des Waffenstillstands die Sicherheit Israels

90 Vgl. Deutscher Bundestag, Stenografischer Bericht, Plenarprotokoll 16/49 vom 19. September, Berlin 2006, S. $4807 \mathrm{f}$.

91 Vgl. Ralf Beste / Horand Knaup / Roland Nelles / René Pfister, Das große Zaudern, in: Der Spiegel vom 7. August 2006, S. $30-32$, S. 32.

92 „Olmerts Wunsch nach deutscher Beteiligung stößt auf Zurückhaltung“, in: DPA-AFX vom 4. August 2006.

93 Hans-Jügen Leersch, Streit um deutschen Einsatz in Nahost, in: Die Welt vom 26. Juli 2006, S. 1.

94 „Lamers: Nahost-Einsatz wird ,kein Spaziergang““, in: DPA-AFX vom 17. August 2006.

95 Hans-Jürgen Leersch, Noch kein Konzept für Friedenstruppe, in: Die Welt vom 27. Juli 2006, S. 2. 
verbessern würde, dann kann man sich dem gerade aus historischer Verantwortung für Israel nicht entziehen." "96 Auch der außenpolitische Sprecher der CDU-Fraktion, Eckart von Klaeden, erachtete die deutsche Geschichte nicht als Hindernis für eine Beteiligung der Bundeswehr an einer Friedensmission. Er vertrat vielmehr die Auffassung, dass „Deutschland seine Pflicht zu tun "97 habe, wenn eine solche Truppe der Herstellung von Frieden dienen könne. Nach der Erweiterung des UNIFIL-Mandats durch Resolution 1701 betonte er entsprechend die internationale Verantwortung Deutschlands: „Wenn es zu einer Lösung kommen soll, dann darf sie sicherlich nicht an Deutschland scheitern. "98

Die internationale Verantwortung Deutschlands wurde auch von anderen Außenpolitikern als ein Argument für einen Einsatz der Bundeswehr angeführt. So begründete beispielsweise der Obmann der CDU/CSU-Fraktion im Auswärtigen Ausschuss, Karl-Theodor Freiherr zu Guttenberg (CSU), seine Offenheit für einen Einsatz mit der Stärkung der internationalen Gemeinschaft. ${ }^{99}$ Ähnlich argumentierte der außenpolitische Sprecher der SPDFraktion, Gert Weisskirchen. Dieser betonte vor allem die Verantwortung des Nahostquartetts (VN, USA, Russland und EU), das für die Herstellung von Sicherheit sorgen und hierfür eine Legitimierung durch den VN-Sicherheitsrat einholen müsse. Sofern dies geschehe, könne sich Deutschland seiner Verantwortung nur schwerlich entziehen. ${ }^{100}$

\section{Fazit: Keine Änderung in Sicht}

Die rechtliche Stellung des Bundestags bei Entscheidungen zur Entsendung der Bundeswehr in Auslandseinsätze ist stark. Es ist jedoch zu bezweifeln, dass sie sich in der politischen Praxis wiederfindet. Aufgrund des geringen Willens von Abgeordneten der Regierungsfraktionen, ihre Widerstände gegen einen Einsatz in Abstimmungen zum Ausdruck zu bringen, der fehlenden Einigkeit unter den Oppositionsfraktionen, infolge derer kein nennenswerter Druck auf die Bundesregierung ausgeübt werden kann, sowie von Unstimmigkeiten unter Fachpolitikern, die ebenfalls eine klare inhaltliche Positionierung des Parlaments verhindern, verliert der Bundestag an Gewicht. Neben den Einschränkungen, die sich aus den Kompetenzen der Bundesregierung ergeben, führen die genannten Aspekte zu zusätzlichen Begrenzungen der Einflussmöglichkeiten des Parlaments.

Exemplarisch ließen ich diese Begrenzungen anhand der Entscheidung zur Teilnahme der Bundeswehr an der VN-geführten Mission UNIFIL im Jahr 2006 zeigen. Es gab deutliche Widerstände innerhalb der Regierungsfraktionen, die sich jedoch nicht im Abstimmungsverhalten der Abgeordneten widerspiegelten. Die Oppositionsfraktionen waren erneut uneins. Während die Grünen mehrheitlich für den Regierungsantrag stimmten, stellten sich die Fraktionen von FDP und Die Linke dagegen. Hinzu kamen fraktionsübergreifende inhaltliche Unstimmigkeiten zwischen Fachpolitikern. Während Verteidigungs-

96 „Streit um Bundeswehreinsatz“, in: DDP vom 25. Juli 2006.

97 „Unionsfraktion noch nicht einig über Beteiligung an Nahost-Einsatz“, in: DDP vom 27. Juli 2006.

98 „Deutsche Truppen in den Nahen Osten“, in: Die Welt vom 16. August 2006, S. 1.

99 Vgl. Ansgar Graw, Deutsche Soldaten für Friedensmission im Nahen Osten?, in: Die Welt vom 19. Juli 2006, S. 5.

100 Vgl. ebenda. 
politiker dem Einsatz eher skeptisch gegenüberstanden, zeigten sich Außenpolitiker deutlich offener.

Dieser Aspekt der Uneinigkeit unter Fachpolitikern ist ein erster Ansatzpunkt für die künftige Forschung. Zu fragen wäre beispielsweise, ob die angeführte Bruchlinie zwischen Außenpolitikern auf der einen und Verteidigungspolitikern auf der anderen Seite auch in anderen außen- und sicherheitspolitischen Kontexten (zum Beispiel in Fragen der europäischen Integration oder in den Beziehungen Deutschlands zu aufstrebenden Mächten) anzutreffen ist. Ein anderer Ansatzpunkt resultiert aus der in diesem Beitrag zur Illustration herangezogenen Entscheidung. Welche Rolle spielen die Besonderheiten einer Großen Koalition im Vergleich zu einer für den deutschen Kontext „typischeren“ Koalitionskonstellation aus einer großen und einer kleinen Partei? Befördern oder hemmen sie die festgestellten Handlungsbegrenzungen, oder haben sie überhaupt keinen Einfluss auf deren Auftreten? Ebenfalls zu untersuchen wäre, welchen Einfluss das vor dem Hintergrund der deutschen Geschichte besonders brisante Einsatzgebiet im Nahen Osten auf die Haltung der Parlamentarier hatte. ${ }^{101}$

Allgemeiner ist außerdem zu fragen, was die zunehmende Integration der deutschen Streitkräfte in europäische (EU) und transatlantische (NATO) Strukturen für die Mitwirkungsrechte des Bundestags bedeutet, nicht zuletzt mit Blick auf das ParlBG. Damit beschäftigt sich gegenwärtig die „Kommission zur Überprüfung und Sicherung der Parlamentsrechte bei der Mandatierung von Auslandseinsätzen der Bundeswehr "102, deren Einsetzung im März 2014 vom Bundestag beschlossen wurde und die sich im Folgemonat unter dem Vorsitz des früheren Bundesverteidigungsministers Volker Rühe (CDU) konstituierte. Bereits der Name der Kommission weist darauf hin, dass es weniger um den Ausbau der Parlamentsrechte geht, als vielmehr um die Frage, wie bestehende Rechte unter sich veränderndenen Bedingungen gewahrt werden können. Inwieweit die Arbeit der Kommission die Rolle des Bundestags aufwerten oder dazu beitragen wird, die in diesem Beitrag angeführten Handlungsbegrenzungen zu überwinden, bleibt abzuwarten.

Eine letzte Frage lautet, ob Parlamente anderer europäischer Staaten in einer ähnlichen Lage sind. $\mathrm{Zu}$ untersuchen wäre insbesondere, ob auch dort, wo die parlamentarischen „war powers“ als „stark“ gelten, die Abgeordneten ihre Rechte ebenfalls nur bedingt wahrnehmen können beziehungsweise wollen. Wenn selbst die vermeintlich starken Parlamente letztlich politisch schwach sind, würde dies eine weitere aus demokratietheoretischer Sicht problematische Komponente zu den von Peters und Wagner konstatierten Befunden hinzufügen: Danach ist es seit Ende des Ost-West-Konflikts tendenziell nicht nur zu keiner „parliamentarisation of war powers " 103 gekommen, sondern Änderungen in dieser Sachfrage sind vorrangig zu Lasten der parlamentarischen Mitspracherechte gegangen.

101 Auch wenn das Einsatzgebiet zweifelsohne von besonderer Brisanz war, sind die in diesem Beitrag angeführten Handlungsbegrenzungen, wie die Ausführungen in den Abschnitten 2.1. bis 2.3. zeigen, nicht spezifisch für UNIFIL. Ob sie bei UNIIFL ausgeprägter waren als in anderen Fällen, kann an dieser Stelle nicht erörtert werden.

102 Vgl. Deutscher Bundestag, Antrag der Fraktionen der CDU/CSU und SPD, Drs. 18/766 vom 11. März, Berlin 2014.

103 Dirk Peters / Wolfgang Wagner, a.a.O. (Fn. 9), S. 185 ff. 